\title{
МОДЕЛИРОВАНИЕ СЦЕНАРИЕВ РАЗВИТИЯ СЕВЕРНЫХ ТЕРРИТОРИЙ: МЕТОДОЛОГИЧЕСКИЙ И ИНФОРМАЦИОННЫЙ АСПЕКТЫ
}

\author{
(c) 2019 Тутыгин Андрей Геннадьевич \\ кандидат физико-математических наук, доцент, ведущий научный сотрудник \\ Федеральный исследовательский центр комплексного изучения Арктики \\ имени академика Н.П. Лаверова РАН, Россия, Архангельск \\ E-mail: andgt64@yandex.ru
}

\section{(c) 2019 Коробов Владимир Борисович}

доктор географических наук, главный научный сотрудник

Федеральный исследовательский центр комплексного изучения Арктики

имени академика Н.П. Лаверова РАН, Россия, Архангельск

директор

Северо-Западное отделение Института океанологии им. П.П. Ширшова РАН, Россия, Архангельск

E-mail:szoioran@mail.ru

\section{(c) 2019 Чижова Людмила Александровна}

кандидат экономических наук, доцент, ведущий научный сотрудник

Федеральный исследовательский центр комплексного изучения Арктики

имени академика Н.П. Лаверова РАН, Россия, Архангельск

E-mail: chijova.mila@yandex.ru

Социо-эколого-экономические системы северных территорий имеют целый ряд специфических проблем и особенностей, которые, с одной стороны, являются существенными при создании сценариев их развития, с другой - с трудом поддаются формализации и количественной оценке при разработке соответствующих моделей. При этом факторы, формирующие особенности северных территорий, крайне разнородны, в том числе, с точки зрения их измеримости и сопоставимости, а информация о них далеко не всегда является полной и аутентичной. Путем соотнесения природно-экологических и социально-экономических факторов, оказывающих существенное влияние на развитие северных территорий, авторы отмечают, что имеющиеся на сегодняшний день информационная база и подходы к моделированию сценариев приводят к определенным системным противоречиям, для разрешения которых требуется создание единого контентного поля. В качестве информационной основы для разработки имитационных моделей предлагается наряду с традиционными наборами статистических показателей использовать оценки, полученные в результате проведения комплексных экспертных процедур. В работе выполнен краткий обзор и сопоставление ряда существующих подходов к разработке моделей динамики развития социо-эколого-экономических систем. В частности, обсуждается проблема статичности внешней среды, которая не может быть преодолена в рамках тех или иных классических подходов. Авторы приходят к выводу о том, что для имитационного моделирования сценариев развития социо-эколого-экономических систем наиболее приемлемым является агент-ориентированный подход.

Ключевые слова: моделирование сценариев развития территорий, социо-эколого-экономические системы, северные территории, технологии экспертных оценок

Исследование выполнено при финансовой поддержке УрО РАН в рамках научного проекта № 18-917-37 «Моделирование эколого-экономических сценариев пространственного развития арктических регионов России».

В «Стратегии пространственного развития Российской Федерации», утвержденной Правительством РФ в феврале 2019 года [1] в России выделено 12 макрорегионов. Один из них - Северный макрорегион, включающий в себя Ар- хангельскую область, Ненецкий автономный округ (НАО) и Республику Коми. Отметим, что при этом территории Архангельской области и Республики Коми - частично, а НАО - полностью, входят в состав Арктической зоны РФ [2]. 
Но здесь нет никакого противоречия, так как соответствующие зонирования территорий отражают различные аспекты их социо-эколого-экономических систем и при этом дополняют друг друга. Поэтому, не ограничивая общности, будем далее такие территории называть северными.

Разделение России на макрорегионы во многом повторяет логику экономического районирования, существовавшую во времена СССР. Так, в 1986 году был выделен Северный экономический район, включавший в себя территории Карельской АССР, Коми АССР, Архангельской (в том числе НАО), Вологодской и Мурманской областей. В нынешней пространственной Стратегии в составе Северного макрорегиона остались Архангельская область, Республика Коми и НАО, территории остальных субъектов РФ отнесены к Северо-Западному макрорегиону.

При этом сохранились такие социально-экономические особенности, характерные для всех регионов, входивших в Северный экономический район, как отраслевая специфика, неразвитая инфраструктура, большие расстояния и высокая доля транспортных расходов в структуре затрат, высокие энергозатраты, повышенная стоимость трудовых ресурсов, низкая плотность населения и отрицательная демографическая динамика. Все это особенно отчетливо проявляется на фоне суровых природных условий и высокой уязвимости экосистем. Следует отметить, что факторы, формирующие особенности северных территорий, крайне разнородны, в том числе, с точки зрения их измеримости и сопоставимости. Имеющаяся и получаемая по ним информация неоднородна по источникам, а зачастую - не аутентична, имеет различную периодизацию и далеко не всегда подвержена количественной формализации. Особенно это относится к факторам, которые относятся к природной среде, экологической ситуации, а также к формированию причинно-следственных связей в демографической ситуации и миграционных процессах.

Информационная база для прогнозирования развития северных территорий с их особой проблематикой на сегодняшний день не представляет собой единый системный контент и не может ограничиваться лишь данными официальной и ведомственной статистики. Нужна адекватная методология моделирования, которая позволит воспроизводить возможные сценарии развития соответствующих социо-эколого-экономиче- ских систем и оценивать их реакцию на те или иные воздействия.

Развитие северных территорий должно базироваться на концепции сбалансированного (устойчивого) развития социо-эколого-экономических систем. Поскольку такие системы отличаются высокой сложностью, рекомендации по их развитию можно разработать только на основании моделирования, для чего необходимо разработать комплекс моделей, имитирующих их функционирование. Учитывая, что такого рода проблемы зачастую не имеют строгого математического решения, необходимо определить содержательные критерии и адекватные им ключевые показатели и индикаторы. При этом отметим, что сбалансированное и устойчивое развитие - все-таки разные понятия. Устойчивое развитие предполагает, что последующие поколения могут получить тот же самый уровень жизни (при этом не учитывается достаточность ресурсов), а сбалансированное - когда рост уровня жизни не ведёт к деградации используемых для этого ресурсов.

Например, как отмечается в работе Р.А. Жукова и др. [3], одной из составляющих устойчивого развития социо-эколого-экономических систем является гармоничное развитие территории, под которым понимается определенное соотношение разномасштабных показателей, имеющих различную природу и размерность. Для его количественной оценки авторы предлагают использовать сформированный из стандартизованных социальных, экологических и экономических индикаторов коэффициент гармоничности

$$
K_{k}=1-\frac{\sigma\left(\xi_{i k}\right)}{M\left(\xi_{i k}\right)}=1-C_{v}\left(\xi_{i k}\right)
$$

близость которого к 1 и означает гармоничность развития. Хоть этот коэффициент и не является бесспорным, его можно использовать в первом приближении для оценки системы в целом. Здесь стандартизованная случайная величина $\xi_{i k}$, соответствующая $i$-тому показателю результативности для $k$-той единицы (объекта) совокупности, получена как

$$
\begin{aligned}
& \xi_{i k}=\frac{\xi_{i k \text { акт }}}{\xi_{\text {kрасч }}} \\
& \xi_{k \text { расч }}=\sqrt{\sum_{i=1}^{m} \sum_{j=1}^{m} \sum_{p=1}^{n} \sum_{q=1}^{n} r_{i j} c_{i p} c_{j q} x_{k p} x_{k q}}
\end{aligned}
$$


где $m$ - число результативных признаков, а $n-$ число факторов, $r_{i j}$ - коэффициент корреляции между соответствующими результативными признаками, $c_{i p}-$ весовой коэффициент $p$-того фактора в формировании $i$-того признака, $x_{k p}$ - фактическое значение стандартизованного $p$-того фактора для $k$-той единицы совокупности.

При проведении модельных расчетов по приведенным формулам кроме статистической информации относительно значений результативных признаков и факторов требуется еще дать оценки весовым коэффициентам влияния (вклада) факторов $c_{i p}$, а эта задача уже находится за рамками корреляционного анализа. Для ее решения предлагается использовать технологию экспертных оценок, например, с применением метода анализа иерархий (МАИ) [4]. Однако, при достаточно большом количестве влияющих факторов и признаков далеко не всегда удается напрямую оценить это влияние. Поэтому следует использовать различные группировки и оценивать как влияние групп факторов, так и внутригрупповые весовые коэффициенты, как это сделано, например, в работах [5, 6]. Другой подход к решению этой задачи - использование метода аналитических сетей, позволяющий оценить косвенные, взаимные и опосредованные связи и, тем самым, уточнить первоначальные весовые коэффициенты [7].

При решении такого рода задач, когда показатели исследуемой системы разнородны, для нахождения весовых коэффициентов, являющихся параметрами модели, их целесообразно группировать по происхождению. В данном случае вполне достаточно все факторы разбить на две группы: социально-экономические и природно-экологические. Это позволяет сформировать группы экспертов в соответствие с их компетенцией. При этом должны выполняться условия:

$$
\begin{aligned}
& \mathrm{c}_{i k} \sum_{1}^{n} c_{i}+c_{j k} \sum_{1}^{m} c_{j}=1 ; \\
& \sum_{1}^{n} c_{i}=1 ; \quad \sum_{1}^{m} c_{j}=1 ; \quad \sum_{1}^{k} c_{i k}=1,
\end{aligned}
$$

где: $c_{i}$ - весовые коэффициенты социальноэкономических факторов, $i=1 \ldots n, n-$ число социально-экономических факторов; $c_{j}$ - весовые коэффициенты природно-экологических факто- ров, $j=1 . . . m, m-$ число природно-экологических факторов; $k=2-$ количество групп. Если количество факторов в группах не совпадает, что обычно бывает на практике, то коэффициенты $c_{i}$ и $c_{j}$ необходимо привести к одному масштабу путём умножения на масштабный множитель $g_{i j}$, рассчитываемый по формуле

$$
g_{i j}=\frac{k \mathrm{c}_{i j}}{n+m} .
$$

Суммирование приведённых по группам весовых коэффициентов позволит выявить наиболее важные из них и оценить соотношение и роли социально-экономических и природно-экологических факторов. Отметим, что для северных территорий это соотношение, как показано авторами в работе [8] на примере формирования нефтяной транспортной инфраструктуры в Арктике, далеко не очевидно.

Таким образом, при разработке сценариев территориального развития необходимо проводить разбиение факторов на группы и оценивать их вклад посредством весовых коэффициентов. При этом процедура экспертного опроса является самостоятельной задачей и проводить её предпочтительнее путём индивидуальных опросов с последующим усреднением результатов [9].

Исходя из имеющегося ресурсного, природно-экологического и социально-экономического потенциала территории, а также с учётом влияния внешних факторов, возможны типовые сценарии развития, приведенные в таблице 1.

Вместе с тем, не исключена возможность комбинации рассмотренных выше сценариев. Например, при благоприятных социально-экономических условиях адаптивный сценарий на определенной инновационной фазе может органично вплестись в инновационную траекторию развития. Вообще, как отмечается в работах $[10,11]$, социально-экономическая динамика не может быть жестко детерминирована, она изначально предполагает альтернативные варианты развития событий в зависимости от того или иного сочетания факторов и ограничений, дает возможность выбора одного из возможных сценариев. Однако это не исключает необходимости разработки стандартных моделей динамики, по которым будут формироваться базовые сценарии развития. Они могут быть приняты за основу при проведении имитационного эксперимента с использованием сценарных матриц. 
Таблица 1. Основные типы сценариев территориального развития

\begin{tabular}{|c|c|}
\hline Тип сценария & Краткая характеристика \\
\hline Инерционный & $\begin{array}{l}\text { Экстенсивное развитие экономики региона в традиционном для нее русле, глав- } \\
\text { ным образом, за счет самостоятельного развития существующих отраслей и про- } \\
\text { изводственных комплексов. Системные проблемы развития территории не могут } \\
\text { быть эффективно решены в обозримой перспективе, регион останется депрессив- } \\
\text { ным, а уровень жизни населения - низким. Сценарий не даст достаточных импуль- } \\
\text { сов для устойчивого социально-экономического развития региона. }\end{array}$ \\
\hline Адаптивный & $\begin{array}{l}\text { Реализация механизмов диверсификации экономики с опорой на малый и средний } \\
\text { бизнес, на предпринимательскую активность. Мобилизация внутренних ресур- } \\
\text { сов, включая определенные ресурсы, высвобождаемые на крупных предприятиях, } \\
\text { ресурсы населения. }\end{array}$ \\
\hline Инновационный & $\begin{array}{l}\text { Предполагает: } \\
\text { - интенсивное развитие экономики, прежде всего, связанное с глубокой перера- } \\
\text { боткой местного сырья; } \\
\text { - развитие научног и кадрового потенциала региона; } \\
\text { - развитие малого и среднего бизнеса, что позволит эффективно решать проблему } \\
\text { занятости населения; } \\
\text { - высокую инфраструктурную обеспеченность территории; } \\
\text { - рост инвестиционной привлекательности региона; } \\
\text { - развитие строительной индустрии, которое позволит обеспечить население } \\
\text { доступным жильем; } \\
\text { - рост реально располагаемых доходов населения; } \\
\text { - развитие социальной сферы на качественно новом уровне. }\end{array}$ \\
\hline Мультипликативный & $\begin{array}{l}\text { Обладая основными чертами инновационного сценария, предполагает развитие } \\
\text { многополярной экономики, предусматривает создание и интенсивное развитие } \\
\text { нескольких региональных экономических кластеров на основе общих системообра- } \\
\text { зующих проектов в стратегической перспективе. Позволяет избежать «монополиза- } \\
\text { ции» региона какой-либо одной отраслью и обеспечит устойчивое развитие эконо- } \\
\text { мики за счет создания взаимоувязанных кластеров с опорой на местные ресурсы. }\end{array}$ \\
\hline
\end{tabular}

Пусть $S=\{1,2, \ldots, m\}-$ множество альтернативных сценариев развития системы, $N=\{1,2, \ldots, n\}-$ множество возможных состояний внешней среды, $a_{i j}(t)$ - динамические оценки ситуации при реализации $i$-того сценария в j-тых условиях, сценарная матрица

$$
A(t)=\left(a_{i j}(t)\right)_{M \times N}
$$

начальные условия - $A(0)$. Описание наборов сценарных траекторий при различных внешних условиях $j=1, \ldots, n$ осуществляется с помощью вектор-функций:

$$
\dot{A}_{j}(t)=\left(\frac{d a_{i j}(t)}{d t}\right)
$$

Ограничения и критерии (аддитивные, мультипликативные, смешанные) при этом конструируются с использованием функций эффективности (полезности) сценариев развития и функций, описывающих потенциальные возможности в рамках состояний внешней среды на определенном временном интервале. Это даёт возможность на основе матричного уравнения разработать соответствующую имитационную модель.

Наиболее краткой, но при этом достаточно ёмкой характеристикой любой модели является её паспорт, который, как минимум, содержит:

- описание номинального объекта (класса объектов) моделирования;

- перечень аспектов рассмотрения объекта;

- цели и задачи, для решения которых строится модель;

- системное описание объекта моделирования;

- описание математического аппарата;

- способы инструментальной и функциональной идентификации;

- способы и границы интерпретации элементов модели.

При этом важнейшим аспектом процесса моделирования является его информационное обеспечение. В структуре информационных массивов различные блоки выделяются, в том числе, по характеру своего содержания и источникам происхождения (таблица 2).

Соотношение различных типов имеющейся, либо добываемой исследователем информации во многом предопределяет выбор того или иного подхода к моделированию проблемной ситуации, объекта или процесса. При этом современные концепции моделирования позволяют 
Таблица 2. Структура информационных массивов процесса моделирования

\begin{tabular}{|l|l|}
\hline \multicolumn{1}{|c|}{ Тип информации } & \multicolumn{1}{c|}{ Источники происхождения } \\
\hline Общетеоретическая & $\begin{array}{l}\text { Научные теории, описывающие классы изучаемых экономических объектов } \\
\text { и процессов }\end{array}$ \\
\hline Предметно-теоретическая & $\begin{array}{l}\text { Предпосылки относительно классов изучаемых объектов и процессов, } \\
\text { принимаемые при построении модели }\end{array}$ \\
\hline Объектная & Значения и оценки показателей моделируемого объекта или процесса \\
\hline Инструментальная & Описание приемов, методов, инструментов моделирования \\
\hline Целевая & Описание целей и задач исследования, сферы применения модели \\
\hline
\end{tabular}

достаточно свободно переносить тот или иной инструментарий, привычный для использования в одних областях знаний, в совершенно другие.

Одним из подходов к моделированию поведения различных сообществ, образующих некоторую систему, является популяционный, давно и широко используемый в естественных науках, в частности, в биологии и в демографии $[12,13]$. Отметим, что под популяцией мы понимаем совокупность особей одного вида, обладающую общим генофондом, способную к более-менее устойчивому самовоспроизводству [14].

Следует упомянуть исторически первую в этом смысле работу Томаса Мальтуса «Опыт о законе народонаселения» [15], написанную ещё в конце XVIII века и успешно использующую аппарат дифференциальных уравнений. Одна из наиболее простых моделей, предложенных Т. Мальтусом, во-первых, исходит из предположения о том, что популяция имеет возможность неограниченно увеличиваться, а во-вторых, что скорость роста пропорциональна её текущей численности. Эти изначальные установки приводят к простейшему дифференциальному уравнению первого порядка:

$$
\frac{d x}{d t}=a x
$$

решением которого будет являться экспонента, описывающая неограниченный рост численности популяции $x(t)$ :

$$
\left\{\begin{array}{c}
x(t)=x_{0} \cdot e^{a t} \\
x_{0}=x(0)
\end{array}\right.
$$

Однако, реалии развития многих сообществ таковы, что модели неограниченного роста могут применяться исследователями лишь на достаточно коротких промежутках времени и со значительными оговорками. Это вполне естественно хотя бы потому, что рост любой по- пуляции ограничен зависимостью от наличия ресурсов, необходимых для её существования. Введение этого условия в модель популяционной динамики привело к логистическому уравнению П. Ферхюльста с асимптотой $K$, ограничивающей сверху численность популяции:

$$
\frac{d x}{d t}=\frac{a \cdot(K-x)}{K},
$$

решение которого также известно в экономикоматематическом моделировании как кривая Перла-Рида:

$$
x(t)=\frac{K}{1+\alpha \cdot e^{-\beta t}}
$$

Известным примером использования логистической кривой является предложенная в 1965 году Г. Тинтнером модель роста народонаселения Швеции, построенная на данных столетнего периода с 1850 по 1950 гг.:

$$
x(t)=\frac{10328806}{1+2,117 \cdot e^{-0,14 t}}
$$

С учётом того, что спустя 40 лет после создания этой модели, в 2005 году, население Швеции составляло порядка 9 млн. человек, это говорит о достаточно высоком её качестве, в том числе, при выборе асимптоты в 10,3 млн. человек.

Отметим, что логистическая кривая относится к классу $S$-образных асимптотически ограниченных кривых, другим известным представителем которого является кривая Б. Гомперца:

$$
x(t)=K \cdot a^{b^{t}},
$$

также описывающая некоторые известные социально-экономические процессы. Так, в 1986 году К. Льюисом была предложена следующая модель расчёта затрат на строительство автомобильных дорог:

$$
I=4644,5 \cdot 0,0961435^{0,93176^{t}}
$$


Вместе с тем, приведённые выше примеры моделей относятся к математико-статистическим, или эконометрическим, а их параметры рассчитываются с использованием различных вариаций метода наименьших квадратов на основе достаточно больших по объему статистических выборок. В какой-то мере эконометрическое моделирование тем самым позволяет использовать популяционный принцип при решении целого ряда экономических задач, имеющих объёмную информационную базу.

Однако, существенной проблемой при моделировании популяционной динамики является то, что популяции зачастую могут существовать не независимо друг от друга, а только при взаимодействии между собой. Наиболее ярким примером здесь является серия моделей типа «хищник-жертва», появившихся в 1920-е годы и известных как модели Лотки-Вольтерра [16, 17], впоследствии также получившие качественное продолжение в работах академика А.Н. Колмогорова [18].

Вместе с тем, несмотря на широкий круг задач, решаемых на основе вышеперечисленных модельных подходов, все они имеют одно очень важное ограничительное условие, принимаемое исследователями «по умолчанию». Оно заключается в том, что внешняя среда принимается неизменной, либо остается таковой в течение достаточно продолжительного времени, что находит отражение в модели как введение некоторых констант. Если же изменения внешней среды всё же учитываются в модели, то это носит, скорее, феноменологический характер, когда объяснение причин влияния внешних факторов остается «за скобками» исследования [19]. Понятно, что для задач социально-экономического плана, решаемых в условиях динамично изменяющейся внешней среды, такое постулирование далеко не всегда является приемлемым. И здесь хотелось бы апеллировать к предложенной Г.Б. Клейнером [20] теории типов систем, в которой выделяются подсистемы средового, объектного, процессного и проектного типов. Данная классификация основана на ограниченности параметров пространства и/или времени (таблица 3).
При этом только при моделировании подсистем проектного типа, и то с очень большими оговорками, условие постоянства внешней среды может быть принято. В подсистемах процессного, объектного и средового типа данная предпосылка зачастую не применима.

Другим аспектом, ограничивающим использование популяционного подхода в моделировании социально-экономического поведения является то, что зачастую соответствующие сообщества не обладают той массовостью, которая характерна для популяций биологического происхождения. Это накладывает определенные ограничения, которые необходимо учитывать в процессе создания моделей поведения, особенно для малых групп, где влияние одного или нескольких участников может иметь решающее значение для дальнейшего развития ситуации. Для отдельных типов сообществ и их поведения решение этой проблемы было предложено, например, М. Грановеттером [21].

И, наконец, в математико-статистических моделях, используемых при изучении поведения популяций, так или иначе предполагается известным, хотя бы гипотетически, тот или иной закон распределения соответствующей случайной величины, а уже затем происходит оценка его параметров. Однако, для тех сообществ, о которых идёт речь в настоящей работе, доля неопределенности в их поведении настолько высока, что применение вероятностно-статистических оценок зачастую оказывается неприемлемым. Объясняется это тем, что в классическом популяционном подходе, говоря биологическим языком, все особи - одинаковы, поэтому поведение каждой из них в отдельности не рассматривается, а лишь принимается как некоторое осреднение общего закона динамики. Если же особенности поведения каждого субъекта сообщества имеют существенное значение, то этот посыл должен быть каким-то образом учтён при создании модели. Это возможно сделать, например, полагая, что каждый член сообщества имеет свои отличия и является так называемым агентом. Соответствующий подход носит название агент-ориентированного моделирования и

Таблица 3. Классификация типов систем по Г.Б. Клейнеру

\begin{tabular}{|c|c|c|c|}
\hline & & \multicolumn{2}{|c|}{ Во времени } \\
\hline & & Ограничена & Не ограничена \\
\hline \multirow{2}{*}{ В пространстве } & Ограничена & Проектный тип & Процессный тип \\
\cline { 2 - 4 } & Не ограничена & Объектный тип & Средовой тип \\
\hline
\end{tabular}


в последнее время получил достаточно широкое распространение при решении различных классов задач. Агент-ориентированные модели, являясь вполне естественным продолжением класса имитационных моделей, обладают следующими основными свойствами [22]:

1. Автономия. Агенты действуют независимо друг от друга и при этом предполагается, что в моделях нет единой регулирующей структуры, которая контролировала бы поведение каждого агента в отдельности. Однако при этом взаимодействие микро- и макроуровней в моделях осуществляется, как правило, следующим образом: на макроуровне задается общий для всех агентов набор правил, и, в свою очередь, совокупность действий агентов микроуровня может оказывать влияние на параметры макроуровня.

2. Неоднородность. Агенты чем-то различаются друг от друга, что принципиально отличает АОМ от широко распространенных моделей с агентом представителем, причем различия между агентами могут проявляться по многим параметрам (в случае агентов, отображающих людей, это могут быть параметры уровня здоровья, дохода, культурного уровня, а также правил принятия решений и т.д.).

3. Ограниченная интеллектуальность агентов (или ограниченная рациональность). Иными словами, агенты модели не могут познать нечто большее, выходящее за рамки макросреды модели.

4. Расположение в пространстве. Имеется в виду некоторая «среда обитания», которая может быть представлена как в виде решетки, так и в виде гораздо более сложной структуры (скажем, трехмерного пространства с заданными в нем объектами).

Согласно перечисленным свойствам агент в АОМ является автономной сущностью, как правило, имеющей графическое представление, с определенной целью функционирования и возможностью обучения в процессе существования до определенного уровня, определяемого разработчиками соответствующей модели. Примерами агентов могут быть:

1) люди (равно как и другие живые организмы), роботы, автомобили и другие подвижные объекты;

2) недвижимые объекты;

3) совокупности однотипных объектов.

Так, агентами в АОМ могут быть любые наблюдаемые в реальной жизни объекты, однако основной задачей их учета в рамках модели является их корректная спецификация.

Общей особенностью всех АОМ и одновременно с этим их главным отличием от моделей других классов является наличие в них большого числа взаимодействующих друг с другом агентов (так, существуют АОМ, число агентов в которых достигает нескольких миллионов).

Обычно в моделях социально-экономических систем присутствуют агрегированные агенты, представляющие собой либо отрасль, либо регион, либо совокупное домохозяйство. При этом спецификация агента происходит за счет оптимизации соответствующей функции полезности или же в модель включаются рассчитанные ранее, в том числе, статистическими методами, экзогенные параметры, отражающие результаты решений агента.

Интересной, с точки зрения применения популяционного и агент-ориентированного подходов к моделированию социально-экономических процессов, является научно-исследовательская работа Ф.А. Белоусова, в которой предложена не имеющая аналогов популяционная модель, учитывающая сложные пространственные ограничения на перемещения агентов по ареалу. Для этой модели получены условия, как сосуществования различных видов агентов, так и вымирания одного из видов. Также автором предложена интерпретация одной из представленных моделей, основанная на отождествлении агентов с экономическими субъектами (фирмами, домохозяйствами и пр.). Подтверждена гипотеза о возможных механизмах возникновения кризисных явлений в экономических системах в результате действия резонанса между поведением различных экономических агентов [23].

В работе В.И. Абрамова приведен пример применения агент-ориентированного подхода к моделированию миграционных процессов. Автор отмечает такие преимущества АОМ как:

- возможность учета явлений, не имеющих явного аналитического представления;

- возможность объяснения нелинейных и сложных взаимодействий, включающих трудно формализуемые элементы [24].

Таким образом, АОМ выступает эффективным инструментом для анализа социально-экономических явлений и процессов, имеющих поведенческий аспект (например, социальный контекст, сеть межличностных отношений и т.д.). 
АОМ является гибким инструментом, позволяющим легко добавлять и удалять агентов в модели, а также менять параметры и правила их поведения. Сегодня в современной науке проводятся многочисленные эксперименты с использованием АОМ, воспроизводящие возможные сценарии развития социально-экономической системы и оценивающие ее реакцию на те или иные управленческие воздействия [25].

Ввиду вышесказанного можно сделать вполне обоснованное предположение о том, что на сегодняшний день АОМ с учетом сказанного выше концептуально является наиболее подходящей методологией для моделирования динамики в социо-эколого-экономических системах.

Выводы:

Описание сценарных условий социо-эколого-экономических систем северных территорий, особенно в части поведения различных сообществ, сталкивается с определенным спектром проблем информационного характера, которые не могут быть преодолены только лишь за счет усовершенствования используемых статистических подходов и методик.

Определенные трудности, возникающие при формировании синтетических критериев принятия решений в условиях неочевидности влияния различных групп факторов, в определенной степени могут быть преодолены за счет использования экспертных технологий, адаптированных к специфическим проблемам и особенностям территорий.

Для имитационного моделирования сценариев развития социо-эколого-экономических систем наиболее приемлемым, на наш взгляд, является агент-ориентированный подход, использующий в качестве информационной основы, с одной стороны, результаты вычислений, полученных классическими математико-статистическими методами, с другой - технологии экспертных оценок.

\section{Библиографический список}

1. Стратегия пространственного развития Российской Федерации (утверждена распоряжением Правительства Российской Федерации 13 февраля 2019 года № 207-р)

2. Указ Президента Российской Федерации «О сухопутных территориях Арктической зоны Российской Федерации» № 296 от 02 мая 2014 года

3. Жуков Р.А., Кузнецов Г.В., Манохин Е.В. К вопросу о формировании критерия оценки гармоничного развития территорий // Современная математика и концепции инновационного математического образования. 2016. T. 3. № 1. С. 151-155.

4. Коробов В.Б., Тутыгин А.Г. Классификационные методы решения эколого-экономических задач: монография / Архангельск: Издательство Поморского государственного университета имени М.В. Ломоносова, 2010.- $310 \mathrm{c}$.

5. Шеломенцев А.Г., Уханова А.В., Смиренникова Е.В., Воронина Л.В. Оценка пространственного развития регионов Арктической зоны Российской Федерации // Региональная экономика и управление: электронный научный журнал. 2018. № 4 (56). Режим доступа: https://eee-region.ru/article/5613/

6. Матвиенко И.И., Тутыгин А.Г., Чижова Л.А. Инвестиционная привлекательность муниципальных образований региона: монография / Архангельск: КИРА, 2012. - 248 с.

7. Середкин К.А., Коробов В.Б., Тутыгин А.Г. О применении метода аналитических сетей в задачах геоэкологии // Проблемы региональной экологии. 2012. № 4. С. 61-65.

8. Тутыгин А.Г., Коробов В.Б. Неопределенность соотношения факторов в задачах принятия решений при строительстве нефтяной транспортной инфраструктуры в Арктике // Экономический рост, ресурсозависимость и социально-экономическое неравенство: Материалы VI Всероссийской конференции 25-27 октября 2018 года. СПб.: Нестор-История, 2018. С. 217-219.

9. Коробов В.Б. Теория и практика экспертных методов: монография.-М.: ИНФРА-М, 2019. - 281 с.

10. Кузык Б.Н., Яковец Ю.В. Интегральный макропрогноз инновационно-технологической и структурной динамики экономики России на период до 2030 года.- М.: Институт экономических стратегий, 2006. - 432 с.

11. Тутыгин А.Г., Чижова Л.А. Методологический подход к формированию инвестиционных сценариев развития экономических систем // Экономика и предпринимательство. 2015. № 10-2 (63). С. 66-69.

12. Базыкин А.Д. Нелинейная динамика взаимодействующих популяций. Москва-Ижевск: Институт компьютерных исследований. 2003.- 368c.

13. Воробьева Т.В., Лаходынова Н.В. О популяционном подходе к моделированию экономических процессов // Экономика и предпринимательство. 2016. № 11-2 (76). С. 1064-1067. 
14. Иоганнсен В.Л. О наследовании в популяциях и чистых линиях / под общей редакцией академика Н.И. Вавилова, ОГИЗ-СЕЛЬХОЗГИЗ, Москва Ленинград, 1935.- 77 с.

15. Мальтус Т.Р. Опыт о законе народонаселения. СПб. 1895.

16. Lotka A.J. Elements of physical biology. Baltimore: Williams and Wilkins, $1925 .-460 \mathrm{p}$.

17. Lotka A. J. Elements of mathimatical biology. New York: Dover Publications, 1956. - 515 p.

18. Колмогоров А.Н. Качественное изучение математических моделей динамики популяций // Проблемы кибернетики, М.: Наука, 1972, вып. 25, С. 100-106.

19. Гумеров М.Ф. Феноменологическая модель распределения и движения информации в системах организационного менеджмента // Управленческие науки. 2017. № 1. С. 75-84.

20. Клейнер Г.Б. Эволюция институциональных систем. М.: Наука, 2004. - 240 с.

21. Granovetter M. S. The strength of weak ties. American Journal of Psychology, 1973. Vol. 78 (6), P. 1360-1380.

22. Ахмедьянова Г.Ф., Ерошенко О.С., Пищухин А.М. Агент-ориентированный подход к моделированию процесса обучения // Фундаментальные исследования. 2013. № 11-3. С. 521-524.

23. Белоусов Ф.А. Модели социально-экономических и демографических процессов сообществ с простейшей социальной структурой / автореферат диссертации на соискание ученой степени кандидата экономических наук, ФГБУН «Центральный экономико-математический институт РАН», Москва, 2019.- 24 с.

24. Абрамов В.И. Опыт применения агент-ориентированного подхода к моделированию процессов в области миграционной политики // Искусственные общества. 2019. Т. 14. Выпуск 2 [Электронный pecypc]. https:// artsoc.jes.su/s207751800005912-2-1. DOI:10.18254/S207751800005912-2

25. Макаров В.Л., Бахтизин А.Р., Сушко Е.Д. Агент-ориентированные модели как инструмент апробации управленческих решений // Управленческое консультирование. 2016. № 12 (96). С. 16-25. 